\title{
Microvascular density under magnifying narrow-band imaging endoscopy in colorectal epithelial neoplasms
}

\author{
Takahiro Gonai, ${ }_{1}^{1}$ Keisuke Kawasaki, ${ }^{1}$ Shotaro Nakamura, ${ }^{1}$ Shunichi Yanai, ${ }^{1}$ Risaburo Akasaka, ${ }_{1}^{1}$ Kunihiko Sato, ${ }^{1}$ \\ Yousuke Toya, ${ }_{1}^{1}$ Kensuke Asakura, ${ }^{1}$ Jun Urushikubo, ${ }^{1}$ Yasuko Fujita, ${ }^{2}$ Makoto Eizuka, ${ }^{2}$ Noriyuki Uesugi, ${ }^{2}$ \\ Tamotsu Sugai, ${ }^{2}$ Takayuki Matsumoto ${ }^{1}$ \\ ${ }^{I}$ Division of Gastroenterology, Department of Internal Medicine, and ${ }^{2}$ Division of Molecular Diagnostic Pathology, Department of Pathology, \\ Iwate Medical University School of Medicine, Morioka, Japan
}

Background/Aims: Magnifying endoscopic classification systems, such as the Japan narrow-band imaging (NBI) Expert Team (JNET) classification, have been widely used for predicting the histologic diagnosis and invasion depth of colorectal epithelial tumors. However, disagreement exists among observers regarding magnifying endoscopic diagnosis, because these classification systems are subjective. We herein investigated the utility of endoscopic microvascular density (eMVD) calculated from magnifying NBI endoscopic images in colorectal tumors. Methods: We reviewed magnifying NBI endoscopic images from 169 colorectal epithelial tumors (97 adenomas, 72 carcinomas/high-grade dysplasias) resected endoscopically or surgically. The eMVD on magnifying NBI endoscopic images was evaluated using image-editing software, and relationships between eMVD and clinical, endoscopic, and pathological findings were retrospectively analyzed. Results: The eMVD in carcinomas $(0.152 \pm 0.079)$ was significantly higher than that in adenomas $(0.119 \pm 0.059, P<0.05)$. The best cutoff value for distinguishing carcinoma from adenoma was 0.133 . Sensitivity, specificity, and accuracy were $56.9 \%, 67.0 \%$, and $62.7 \%$, respectively. In addition, JNET type $2 \mathrm{~B}$ tumors showed significantly higher eMVD $(0.162 \pm 0.079)$ compared to type $2 \mathrm{~A}$ tumors $(0.111 \pm 0.050$, $P<0.05)$. Conclusions: The eMVD as determined by magnifying NBI endoscopy is considered to be a possible objective indicator for differentiating colorectal carcinomas from adenomas. (Intest Res 2020;18:107-114)

Key Words: Microvascular density; Narrow band imaging; Colorectal neoplasms; Colonoscopy

\section{INTRODUCTION}

Magnifying endoscopy has become one of the most useful modalities for detailed histologic diagnosis of colorectal neoplastic lesions. Notably, narrow-band imaging (NBI) magnifying endoscopy has contributed greatly to the optical diagnosis of GI lesions by enabling clear visualization of vascular architecture and surface structure, ${ }^{1}$ and has been widely adopted in Japan. Since it was established in 2011, ${ }^{2}$ the Japan NBI Ex-

Received May 21, 2019. Revised August 7, 2019. Accepted September 6, 2019. Correspondence to Takahiro Gonai, Division of Gastroenterology, Department of Internal Medicine, Iwate Medical University School of Medicine, Uchimaru 19-1, Morioka 020-8505, Japan. Tel: +81-19-651-5111,

Fax: +81-19-652-6664, E-mail: tgonai@iwate-med.ac.jp pert Team (JNET) classification has been commonly used to predict histological structures and invasion depth of colorectal epithelial neoplasms. However, some disagreement exists among observers regarding diagnosis using the JNET classification, because it is subjective in nature. To date, only a few groups have reported objective indicators for evaluating colorectal epithelial tumors. ${ }^{3}$ According to the JNET classification, low-grade intramucosal neoplasia (adenoma or carcinoma with low-grade structural atypia) is classified as type $2 \mathrm{~A}$, which shows a uniform regular, relatively well-ordered reticular vascular pattern and a tubular or papillary regular surface pattern, while high-grade intramucosal neoplasia or shallow submucosal ( $\mathrm{sSM}$ ) invasive cancer is classified as type $2 \mathrm{~B}$, which shows varied caliber, thick or dilated vessels and a variable, irregular 
surface pattern. In contrast, deep submucosal (dSM) invasive cancer is classified as type 3 , which shows avascular or loose vascular areas or disrupted thick vessels and an invisible amorphous surface pattern. ${ }^{4,5}$ In addition, other reports have suggested that the microvascular structure differs depending on the histological type of colon tumor. ${ }^{6,7}$ Based on these findings, we speculate that tumors with higher microvascular density (MVD) should tend to have higher-grade histology and deeper intramural invasion.

In the present study, we calculated MVD in colorectal epithelial tumors using image processing software to clarify the correlation between MVD and clinicopathological findings.

\section{METHODS}

\section{Subjects}

From 2014 to 2016, a total of 169 colorectal epithelial tumors (97 adenomas and 72 carcinomas or high-grade dysplasias [HGD]) in 153 patients that were evaluated by magnifying NBI endoscopy (CF-H260AZ or CF-H290Z; Olympus, Tokyo, Japan) and were resected by endoscopic mucosal resection, endoscopic submucosal dissection, or surgery were included. The present study protocol was approved by the Institutional Review Board at Iwate Medical University (IRB No. MH2018050), and the study was conducted in accordance with the Helsinki Declaration (6th revision, 2008). Written informed consents were obtained.

\section{Colonoscopic Assessment}

All endoscopic images from the 169 colorectal tumors acquired under conventional and magnifying observation were retrospectively evaluated. The location of each lesion was classified as right side (cecum to transverse colon) or left side (descending colon to rectum). The gross morphology of tumors was classified as either protruded type or flat-elevated type, according to the Paris classification. ${ }^{8}$ White opaque substance (WOS) was defined as a whitish area under magnifying NBI endoscopy, which obscures the microvascular pattern within the colorectal epithelial neoplasm. ${ }^{9}$

All 169 tumors were retrospectively reclassified either as type 1, 2, or 3 according to the NBI International Colorectal Endoscopic (NICE) non-magnifying classification, ${ }^{10}$ and as type $2 \mathrm{~A}, 2 \mathrm{~B}$, or 3 according to the JNET classification ${ }^{2}$ by 2 endoscopists (T.G. and K.K.) who had experienced total colonoscopy in 1,000 and 8,000 cases, respectively.

\section{Histological Analysis}

Two specialized pathologists (T.S. and M.E.), both of whom are experts in histopathologic analysis of colorectal neoplasms, evaluated all 169 resected tumors. In this study, only low-grade dysplasia was classified as adenoma, while HGD was classified as carcinoma. A carcinoma that vertically invaded up to $1,000 \mu \mathrm{m}$ in the submucosal layer was classified as SSM carcinoma, and that which massively invaded the submucosa by $\geq 1,000 \mu \mathrm{m}$ was classified as dSM carcinoma. The grade of tumor budding, lymphatic permeation, and venous invasion were also evaluated.

\section{Endoscopic Microvascular Density Image Analysis} Endoscopic MVD (eMVD) based on endoscopic magnifying NBI images of colorectal tumors was calculated by using the image processing software ImageJ version 1.51j8 (Wayne Rasband National Institutes of Health, Bethesda, MD, USA). We selected the most appropriate NBI magnifying endoscopic image to predict the histological type of each tumor, which was used as the representative image of each tumor. Each image was imported into ImageJ, which split the image into red, green, and blue channels. We used blue channel images, because the contrast between the microsurface and microvessel pattern was visualized more clearly on the blue channel than on the red or green channels. The outer areas of the tumor were cropped from the image, and the shading threshold was adjusted. The threshold was uniformly set to 40 (0-255, 8 bit). By setting the threshold, the images were binarized, microsurface and microvessel pattern were completely separated. Images of miwcrovessels and background mucosa were edited in black and in white, respectively. The eMVD was determined by calculating the proportion of black areas in the entire image (Fig. 1). In this image analysis, image processing took several minutes manually, while calculation of eMVD took only a moment automatically. The eMVD was automatically calculated in a few seconds when image processing is appropriately performed using programming.

\section{Statistical Analysis}

Statistical analysis was performed using JMP for Mac (Statistical Discovery Program, Cary, NC, USA). Since the numeric data for eMVD were non-parametric, comparisons between the 2 groups were performed based on Mann-Whitney $U$-test. A receiver operator characteristic (ROC) graph was drawn to estimate the best cutoff value for differentiating carcinoma from adenoma. According to the cutoff value, test significance 

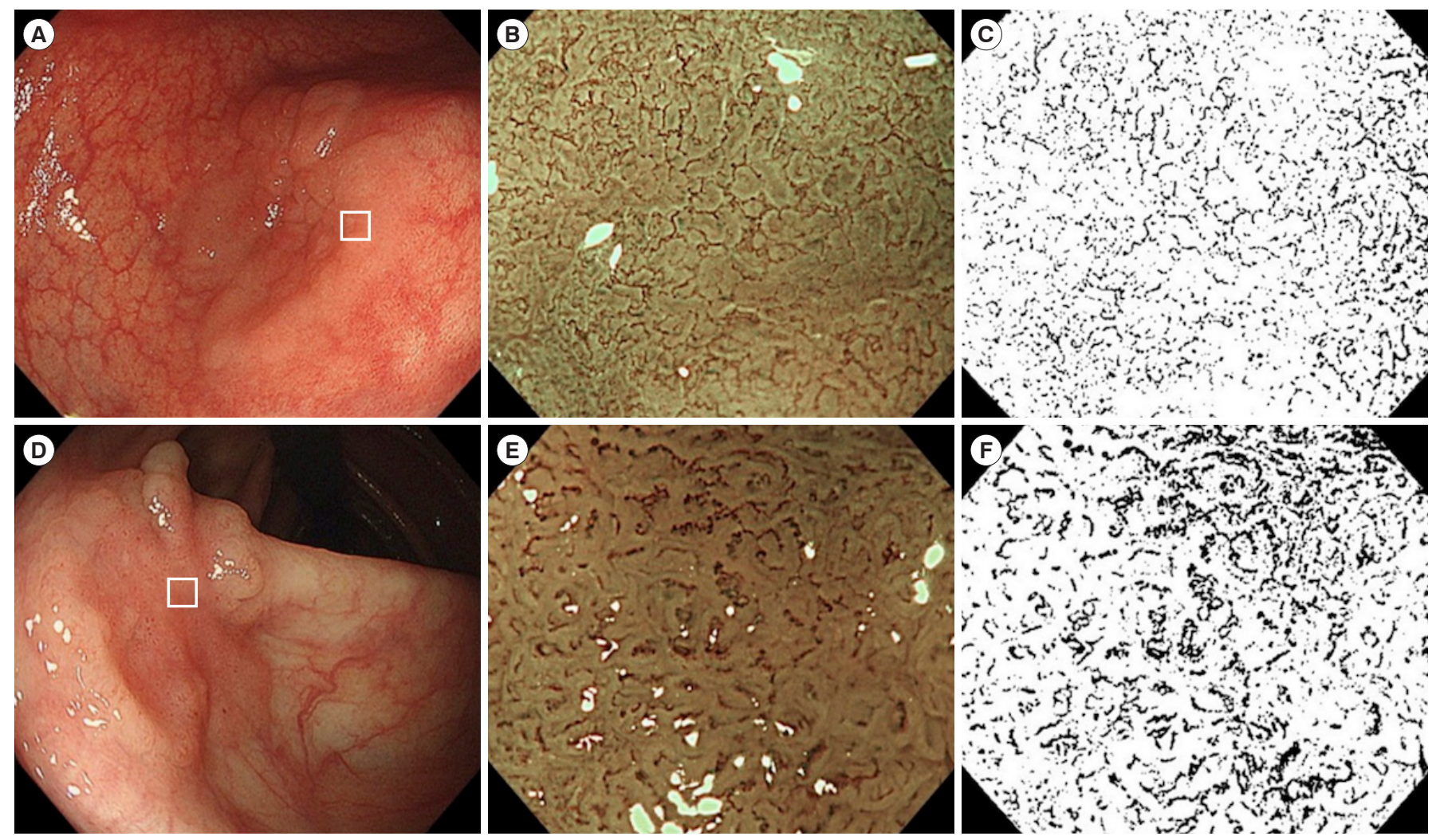

Fig. 1. Examples of white light, magnifying narrow-band imaging (NBI) endoscopic, and magnifying monochrome images. (A) A laterally spreading tumor in the sigmoid colon. A depressed area (white square) is subsequently observed by magnifying endoscopy. (B) A magnifying NBI endoscopic image in the tumor. Microvessels at the surface are thin and uniform. (C) Edited image from (B) using ImageJ (monochrome image). Black vascular areas and white avascular areas can be seen. The calculated endoscopic microvascular density (eMVD) of this image is 0.103 . The histological diagnosis was tubular adenoma. (D) Laterally spreading tumor in the ascending colon. A depressed area (white square) is subsequently observed by magnifying endoscopy. (E) Magnifying NBI endoscopic image of the tumor. Microvessels in the surface are thick and meandering. (F) Edited image from (E) using ImageJ. The calculated eMVD of this image is 0.176. The histological diagnosis was shallow submucosal carcinoma.

including sensitivity, specificity, positive predictive value (PPV), negative predictive value (NPV), and accuracy were calculated. $P$-values $<0.05$ were considered statistically significant.

\section{RESULTS}

A total of 153 patients ( 87 men, 66 women) were enrolled. The mean age was 69 years. Among 14 patients with multiple (up to 3) lesions, the lesion with the highest eMVD was selected as the representative lesion for that patient in analyzing age and sex. Among 153 patients, no significant differences in eMVD were observed with respect to $\operatorname{sex}(0.127 \pm 0.069$ in men, $0.141 \pm$ 0.069 in women $)$ or age $(0.132 \pm 0.068$ in patients $<65$ years, $0.136 \pm 0.072$ in patients $\geq 65$ years).

Table 1 shows the relationship between eMVD and endoscopic and clinicopathological findings in the 169 tumors. The
eMVD in macroscopically protruded type tumors $(0.152 \pm 0.076)$ was higher than that in flat-elevated type tumors $(0.125 \pm 0.064$, $P<0.05)$, while that in carcinomas or HGDs $(0.152 \pm 0.079)$ was higher than that in adenomas $(0.119 \pm 0.059, P<0.05)$. No significant differences were observed with respect to tumor size, location, or WOS. ROC analysis revealed a cutoff value for distinguishing carcinoma/HGD from adenoma of 0.133 (AUC, 0.62; 95\% CI, 0.54-0.71) (Fig. 2). The sensitivity, specificity, and accuracy were $56.9 \%, 67.0 \%$, and $62.7 \%$, respectively.

Among 72 carcinomas or HGDs, no significant differences were observed in eMVD with respect to the depth of tumor invasion, histologic type, tumor budding, lymphatic permeation, or venous invasion, as shown in Table 2. In adenomas, there was no significant difference in eMVD between tubular adenomas and tubulovillous adenomas (Table 3). Cases of villous adenoma were not included in the present study. In adeno- 
Table 1. Relationship between eMVD and Endoscopic and Clinicopathological Findings of 169 Colorectal Tumors

\begin{tabular}{|c|c|c|c|}
\hline Variable & No. (\%) & $\begin{array}{c}\text { eMVD, } \\
\text { mean } \pm \text { SD }\end{array}$ & $P$-value \\
\hline Size $(m m)^{a}$ & & & 0.707 \\
\hline$\leq 20$ & $56(33)$ & $0.139 \pm 0.073$ & \\
\hline$>20$ & $113(67)$ & $0.130 \pm 0.068$ & \\
\hline Location & & & 0.829 \\
\hline Right colon & $84(50)$ & $0.130 \pm 0.068$ & \\
\hline Left colon or rectum & $85(50)$ & $0.137 \pm 0.071$ & \\
\hline Macroscopic type & & & $<0.050$ \\
\hline Protruded type & $55(33)$ & $0.152 \pm 0.076$ & \\
\hline Flat-elevated type & $114(67)$ & $0.125 \pm 0.064$ & \\
\hline WOS & & & 0.460 \\
\hline Positive & $104(62)$ & $0.131 \pm 0.072$ & \\
\hline Negative & $65(38)$ & $0.137 \pm 0.066$ & \\
\hline Histologic type & & & $<0.050$ \\
\hline Adenoma & $97(57)$ & $0.119 \pm 0.059$ & \\
\hline Carcinoma or HGD & $72(43)$ & $0.152 \pm 0.079$ & \\
\hline
\end{tabular}

${ }^{a}$ The mean \pm SD of the size is $28.0 \pm 17 \mathrm{~mm}$.

eMVD, endoscopic microvascular density; WOS, white opaque substance; HGD, high-grade dysplasia.

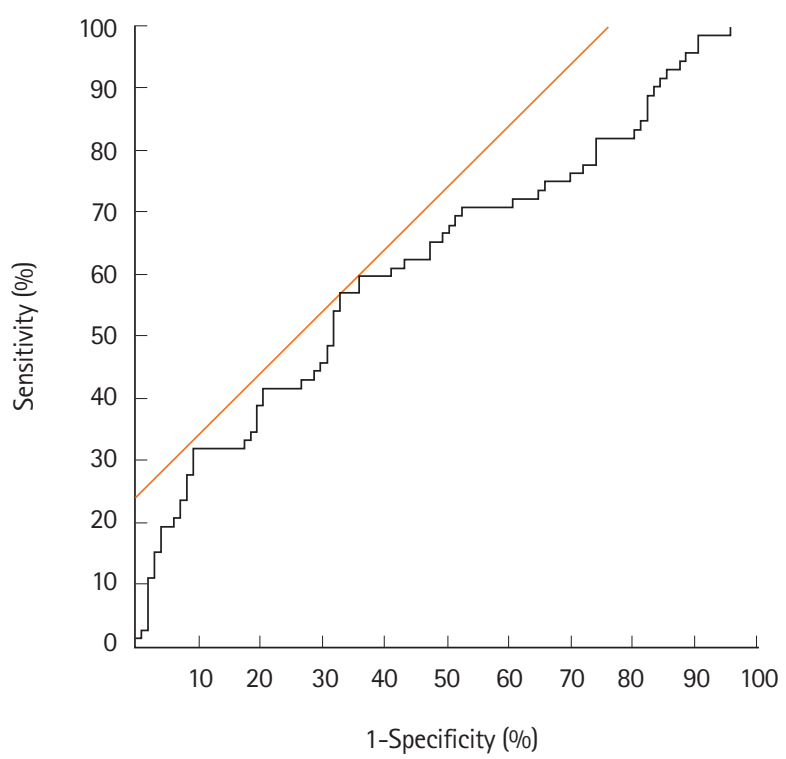

Fig. 2. Receiver operator characteristic (ROC) curve for distinguishing carcinoma/high-grade dysplasia from adenoma. ROC for endoscopic microvascular density to predict histologic carcinoma. The best cutoff value for distinguishing adenoma and carcinoma was calculated to be 0.133 , with a sensitivity of $56.9 \%$, a specificity of $67.0 \%$, and an accuracy of $62.7 \%$.
Table 2. Relationship between eMVD and Histopathological Findings of 72 Colorectal Carcinomas or HGDs

\begin{tabular}{lccc}
\hline Variable & No. (\%) & $\begin{array}{c}\text { eMVD, } \\
\text { mean } \pm \text { SD }\end{array}$ & P-value \\
\hline $\begin{array}{l}\text { Depth of invasion } \\
\text { HGD \& sSM }\end{array}$ & $53(74)$ & $0.154 \pm 0.078$ & 0.565 \\
dSM & $19(26)$ & $0.147 \pm 0.079$ & \\
Histologic type & & & \\
$\quad$ Papillary (pap) & $10(14)$ & $0.150 \pm 0.088$ & $0.818^{\mathrm{a}}$ \\
Well differentiated (tub1) & $26(36)$ & $0.155 \pm 0.081$ & $0.915^{\mathrm{b}}$ \\
Moderately differentiated (tub2) & $36(50)$ & $0.151 \pm 0.075$ & $0.800^{c}$ \\
Budding & & & \\
$\quad$ None & $67(93)$ & $0.152 \pm 0.079$ & $0.582^{\mathrm{d}}$ \\
Grade 1 & $3(4)$ & $0.121 \pm 0.052$ & $0.148^{\mathrm{e}}$ \\
Grade 2 & $2(3)$ & $0.211 \pm 0.019$ & $0.180^{f}$ \\
Lymphatic permeation & & & 0.683 \\
Positive & $9(13)$ & $0.164 \pm 0.088$ & \\
Negative & $63(87)$ & $0.150 \pm 0.077$ & \\
Venous invasion & & & 0.947 \\
Positive & $5(7)$ & $0.145 \pm 0.053$ & \\
Negative & $67(93)$ & $0.153 \pm 0.080$ & \\
\hline
\end{tabular}

apap vs. tub1.

tub1 vs. tub2.

'pap vs. tub2.

${ }^{\mathrm{d}}$ None vs. grade 1.

${ }^{e}$ Grade 1 vs. grade 2.

fNone vs. grade 2.

eMVD, endoscopic microvascular density; HGD, high-grade dysplasia; sSM, shallow submucosa; dSM, deep submucosa.

Table 3. Relationship between eMVD and Histopathological Findings of 97 Colorectal Adenomas

\begin{tabular}{lccc}
\hline & No. (\%) & $\begin{array}{c}\text { eMVD, } \\
\text { mean } \pm \text { SD }\end{array}$ & $P$-value \\
\hline Histologic type & & & 0.972 \\
Tubular adenoma & $66(68)$ & $0.119 \pm 0.053$ & \\
Tubulovillous adenoma & $31(32)$ & $0.122 \pm 0.070$ & \\
\hline
\end{tabular}

eMVD, endoscopic microvascular density.

mas, eMVD did not differ between protruded type and flat-elevated type, while in carcinomas or HGDs, eMVD tended to be higher in protruded type than in flat-elevated type $(P=0.075)$ (Table 4). Furthermore, we have determined a cutoff value for distinguishing carcinoma/HGD from adenoma in protruded tumors to be 0.138 , while that in flat-elevated tumors to be 0.110 .

Table 5 shows the relationship between eMVD and NICE 
Table 4. Relationship between eMVD and Macroscopic Type in Each Histologic Type

\begin{tabular}{lccc}
\hline & No. (\%) & $\begin{array}{c}\text { eMVD, } \\
\text { mean } \pm \text { SD }\end{array}$ & P-value \\
\hline Adenoma & & & 0.675 \\
$\quad$ Protruded type & $24(25)$ & $0.130 \pm 0.067$ & \\
$\quad$ Flat-elevated type & $73(75)$ & $0.116 \pm 0.056$ & \\
$\begin{array}{l}\text { Carcinoma or HGD } \\
\text { Protruded type }\end{array}$ & $31(43)$ & $0.169 \pm 0.080$ & \\
Flat-elevated type & $41(57)$ & $0.140 \pm 0.076$ & \\
\hline
\end{tabular}

eMVD, endoscopic microvascular density; HGD, high-grade dysplasia.

Table 5. Relationship between eMVD and NICE Classification Type, JNET Classification Type in 169 Tumors

\begin{tabular}{lccc}
\hline & No. (\%) & $\begin{array}{c}\text { eMVD, } \\
\text { mean } \pm \text { SD }\end{array}$ & $P$-value \\
\hline NICE classification & $3(2)$ & $0.066 \pm 0.066$ & $0.089^{\mathrm{a}}$ \\
Type 1 & $136(80)$ & $0.135 \pm 0.067$ & $0.664^{\mathrm{b}}$ \\
Type 2 & $30(18)$ & $0.133 \pm 0.078$ & $0.125^{\mathrm{c}}$ \\
Type 3 & & & \\
JNET classification & $88(52)$ & $0.111 \pm 0.050$ & $<0.050^{\mathrm{d}}$ \\
Type 2A & $65(38)$ & $0.162 \pm 0.079$ & $0.283^{\mathrm{e}}$ \\
Type 2B & $16(10)$ & $0.141 \pm 0.077$ & $0.196^{\mathrm{f}}$ \\
\hline Type 3 & & &
\end{tabular}

Type 1 vs. type 2.

${ }^{\text {'} T y p e ~} 2$ vs. type 3.

'Type 1 vs. type 3.

${ }^{\mathrm{D}}$ Type $2 \mathrm{~A}$ vs. type $2 \mathrm{~B}$.

'Type $2 B$ vs. type 3.

'Type 2 A vs. type 3.

eMVD, endoscopic microvascular density; NBI, narrow-band imaging; NICE, NBI International Colorectal Endoscopic; JNET, Japan NBI Expert Team.

classification or JNET classification. In NICE classification, all 169 tumors were classified either as type $1(n=3)$, type $2(n=136)$, or type $3(\mathrm{n}=30)$. Significant differences were not observed between any 2 groups (type 1 vs. type 2, $P=0.089$; type 2 vs. type $3, P=0.664$; type 1 vs. type $3, P=0.125$ ). In JNET classification, all 169 tumors were classified either as type $2 \mathrm{~A}(\mathrm{n}=88)$, type $2 \mathrm{~B}(\mathrm{n}=65)$, or type $3(\mathrm{n}=16)$. The eMVD in type $2 \mathrm{~B}$ tumors $(0.162 \pm 0.079)$ was significantly higher than that in type 2 A tumors $(0.111 \pm 0.050, P<0.05)$ (Fig. 3$)$. No significant difference was observed between type $2 \mathrm{~A}$ and type 3 tumors ( $P=0.196)$, or between type $2 \mathrm{~B}$ and type 3 tumors $(P=0.283)$.

Table 6 summarizes the sensitivity, specificity, PPV, NPV and accuracy for distinguishing carcinoma/HGD from adeno-

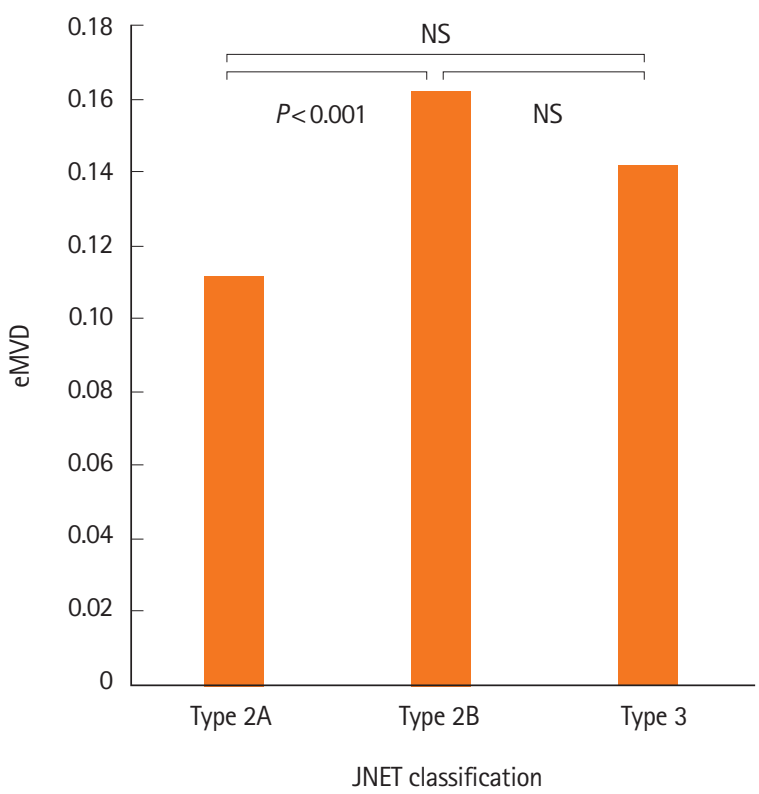

Fig. 3. Endoscopic microvascular density (eMVD) of tumors by JNET classification. Relationship between eMVD and JNET classification. A significant difference in eMVD is observed between JNET type $2 \mathrm{~A}$ and $2 \mathrm{~B}$ tumors $(P<0.001) . \mathrm{NBI}$, narrow-band imaging; JNET, Japan NBI Expert Team.

Table 6. Sensitivity, Specificity, PPV, NPV, and Accuracy of eMVD System and JNET Classification in the Diagnosis of Carcinoma or HGD

\begin{tabular}{lccccc}
\hline Findings & $\begin{array}{c}\text { Sensitivity } \\
(\%)\end{array}$ & $\begin{array}{c}\text { Specificity } \\
(\%)\end{array}$ & $\begin{array}{c}\text { PPV } \\
(\%)\end{array}$ & $\begin{array}{c}\text { NPV } \\
(\%)\end{array}$ & $\begin{array}{c}\text { Accuracy } \\
(\%)\end{array}$ \\
\hline eMVD & 56.9 & 67.0 & 57.7 & 75.6 & 62.7 \\
JNET & 76.4 & 73.2 & 67.9 & 80.1 & 74.6 \\
eMVD+JNET & 87.5 & 53.6 & 58.3 & 85.2 & 68.0 \\
\hline
\end{tabular}

PPV, positive predictive value; NPV, negative predictive value; eMVD, endoscopic microvascular density; NBI, narrow-band imaging; JNET, Japan NBI Expert Team; HGD, high-grade dysplasia.

ma by each of eMVD, JNET classification and the both. Diagnostic activities of eMVD, including sensitivity, specificity, PPV, NPV and accuracy, seemed inferior to JNET classification. When eMVD and JNET classification are combined and the higher grade of dysplasia determined by either procedure was adopted as the representative grade, sensitivity (87.5\%) and NPV (85.2\%) showed highest values among these 3 modalities for the distinction of carcinoma/HGD from adenoma.

\section{DISCUSSION}

In the present study, we objectively evaluated eMVD in colorec- 
tal epithelial tumors using the image processing software ImageJ, which revealed that the eMVD was significantly higher in early colorectal carcinomas/HGDs than in adenomas (Table 1). In addition, JNET type $2 B$ tumors had a significantly higher eMVD than type 2A tumors (Table 5, Fig. 3).

Tumor angiogenesis is important for tumor growth, proliferation, and metastasis. ${ }^{11}$ Vascular endothelial growth factor (VEGF) plays an essential role in tumor angiogenesis. VEGF binds to receptors on endothelial cells, thereby promoting their proliferation and survival. ${ }^{11,12}$ VEGF expression level has been reported to be correlated with histological MVD, ${ }^{12}$ and histological MVD increases as tumor invasion progresses. ${ }^{12-14}$ In addition, histological MVD correlates with prognosis among patients with colorectal cancer. ${ }^{15-17}$ A meta-analysis revealed that high histological MVD is significantly associated with poor relapse-free and overall survivals of patients. ${ }^{16}$

In colorectal tumor progression, angiogenesis begins during a phase called the "angiogenic switch," which is thought to occur at the onset of dysplasia in the adenoma-carcinoma sequence, suggesting that active angiogenesis is initiated during the earliest stage of colorectal tumorigenesis. The histological MVD also significantly increases as the adenoma-carcinoma sequence progresses. ${ }^{12,15}$ In the present study, a significant difference in eMVD was observed between adenomas and carcinomas, which suggests that angiogenesis also progresses throughout the adenoma-carcinoma sequence.

In several studies, the histological MVD was reported to increase with deeper tumor invasion. ${ }^{12-15}$ In our study, however, eMVD did not differ between HGD/sSM carcinoma and dSM carcinoma (Table 2, Fig. 3). The insignificant difference is probably because dSM carcinomas or JNET type 3 tumors often show avascular or loose vascular areas, disrupted thick vessels, and an invisible amorphous surface pattern under magnifying NBI endoscopy. We speculate that in dSM carcinomas, increased vascularity should have occurred around the deeper portion of the carcinoma cells, contrasting to less vascularity in the superficial portion. In addition, small number of cases of dSM carcinomas $(n=19)$ might be another reason for the discrepancy.

It is of interest that the mean eMVD in macroscopically protruded type tumors was higher than that in flat-elevated type tumors ( 0.152 vs. $0.125, P<0.05)$ (Table 1). Protruded type tumors tend to have stalked or broad-based microvessel architecture, whereas flat-elevated type tumors tend to have sessile microvessel architecture. ${ }^{18}$ Conversely, the presence of WOS did not affect eMVD (Table 1). WOS has been reported to fre- quently interfere with the visibility of the microvessels, and WOS is more frequently observed in carcinomas than in adenomas. ${ }^{19}$ We thus consider that both factors might have offset the possible influence of WOS on eMVD.

In JNET classification, which was a subjective indicator, the eMVD in type 2B tumors was significantly higher than that in type 2A tumors (Table 5). In NICE classification, however, no significant differences were observed between type 2 and 3 (Table 5). This discrepancy was probably because type 2 tumors in NICE classification include adenomas with low-grade dysplasia or HGD, mucosal carcinomas, and superficial submucosal carcinomas, and might have included type $2 \mathrm{~A}$ and 2B tumors in JNET classification. It should be noted that combination of eMVD plus JNET classification improved sensitivity and NPV in the diagnosis of carcinoma or HGD, as shown in Table 6.

Recently, computer-aided diagnosis (CAD) systems for colorectal lesions, including artificial intelligence-assisted colonoscopy, have been developed. ${ }^{3,20-22}$ Mori et al. ${ }^{20}$ reported on the efficacy of CAD using endocytoscopy (450 × magnification), with a sensitivity of $92 \%$ and an accuracy of $89 \%$. Chen et al. ${ }^{22}$ developed a CAD system using a deep neural network to analyze non-magnifying NBI images of diminutive colorectal polyps, with $96 \%$ sensitivity, $78 \%$ specificity, $89 \%$ PPV, and $91 \%$ NPV. Another CAD system by Tamai et al. ${ }^{3}$ using magnifying NBI images showed $84 \%$ sensitivity, $83 \%$ specificity, 53\% PPV, $96 \%$ NPV, and $83 \%$ accuracy. We expect that a new CAD system using our eMVD method will be developed in the near future.

The present study has several limitations. First, eMVD may be insufficient to distinguish between adenoma and carcinoma, as the accuracy is only $62.7 \%$. It is difficult to diagnose adenoma or carcinoma by eMVD alone. Second, we determined eMVD from capillary vessels using the horizontal surfaces of tumors, while many of the previous studies assessed histological MVD from vertical cut surfaces. This difference might have led to disagreement between endoscopic MVD and histological MVD.

In conclusion, eMVD as determined by magnifying NBI endoscopy is considered to be a possible objective indicator for differentiating colorectal carcinomas from adenomas. While it is insufficient to predict exact histological diagnosis of colorectal neoplasia only by eMVD, combination of eMVD plus JNET classification may be more useful. Further improvement of image evaluation methods is required for accurate histologic diagnosis of colorectal cancers, including dSM carcinomas. 


\section{FINANCIAL SUPPORT}

The authors received no financial support for the research, authorship, and/or publication of this article.

\section{CONFLICT OF INTEREST}

No potential conflict of interest relevant to this article was reported.

\section{AUTHOR CONTRIBUTION}

Conceptualization: Gonai T. Methodology: Kawasaki K, Nakamura S. Validation: Sugai T, Matsumoto T. Formal analysis: Gonai T. Resources: Gonai T, Kawasaki K, Yanai S, Akasaka R, Sato K, Toya Y, Asakura K, Urushikubo J. Data curation: Eizuka M, Fujita Y, Uesugi N, Sugai T. Writing - original draft: Gonai T. Writing - review \& editing: Kawasaki K, Nakamura S, Matsumoto T. Approval of final manuscript: all authors.

\section{ORCID}

Gonai T

Kawasaki K

Nakamura S

Yanai S

Akasaka R

Sato K

Toya $\mathrm{Y}$

Asakura K

Urushikubo J

Fujita Y

Eizuka M

Uesugi $\mathrm{N}$

Sugai $\mathrm{T}$

Matsumoto T https://orcid.org/0000-0001-6759-2459

https://orcid.org/0000-0003-2645-4417 https://orcid.org/0000-0001-8166-2599 https://orcid.org/0000-0003-1871-2412 https://orcid.org/0000-0001-7087-3016 https://orcid.org/0000-0002-0834-3353 https://orcid.org/0000-0002-0990-9304 https://orcid.org/0000-0002-4653-3801 https://orcid.org/0000-0002-4808-7614 https://orcid.org/0000-0002-3988-9076 https://orcid.org/0000-0003-4815-1273 https://orcid.org/0000-0002-4388-6660 https://orcid.org/0000-0002-4896-3557 https://orcid.org/0000-0001-9786-3854

\section{REFERENCES}

1. Yao K, Anagnostopoulos GK, Ragunath K. Magnifying endoscopy for diagnosing and delineating early gastric cancer. Endoscopy 2009;41:462-467.

2. Sano Y, Tanaka S, Kudo SE, et al. Narrow-band imaging (NBI) magnifying endoscopic classification of colorectal tumors proposed by the Japan NBI Expert Team. Dig Endosc 2016;28: 526-533.
3. Tamai N, Saito Y, Sakamoto T, et al. Effectiveness of computeraided diagnosis of colorectal lesions using novel software for magnifying narrow-band imaging: a pilot study. Endosc Int Open 2017;5:E690-E694.

4. Hashimoto R, Matsuda T, Hamamoto H, Yamaoka H, Nakahori M, Chonan A. Usefulness of dilated blood vessels in the tumor periphery for assessing the invasion depth of small-sized depressed colorectal cancer. Medicine (Baltimore) 2016;95: e3913.

5. Hirata M, Tanaka S, Oka S, et al. Evaluation of microvessels in colorectal tumors by narrow band imaging magnification. Gastrointest Endosc 2007;66:945-952.

6. Konerding MA, Fait E, Gaumann A. 3D microvascular architecture of pre-cancerous lesions and invasive carcinomas of the colon. Br J Cancer 2001;84:1354-1362.

7. Mizuno K, Kudo SE, Ohtsuka K, et al. Narrow-banding images and structures of microvessels of colonic lesions. Dig Dis Sci 2011;56:1811-1817.

8. The Paris endoscopic classification of superficial neoplastic lesions: esophagus, stomach, and colon: November 30 to December 1, 2002. Gastrointest Endosc 2003;58(6 Suppl):S3-S43.

9. Kawasaki K, Kurahara K, Yanai S, et al. Significance of a white opaque substance under magnifying narrow-band imaging colonoscopy for the diagnosis of colorectal epithelial neoplasms. Gastrointest Endosc 2015;82:1097-1104.

10. Hayashi N, Tanaka S, Hewett DG, et al. Endoscopic prediction of deep submucosal invasive carcinoma: validation of the narrow-band imaging international colorectal endoscopic (NICE) classification. Gastrointest Endosc 2013;78:625-632.

11. Kerbel RS. Tumor angiogenesis. N Engl J Med 2008;358:20392049.

12. Takahashi Y, Ellis LM, Mai M. The angiogenic switch of human colon cancer occurs simultaneous to initiation of invasion. Oncol Rep 2003;10:9-13.

13. Wali RK, Roy HK, Kim YL, et al. Increased microvascular blood content is an early event in colon carcinogenesis. Gut 2005;54: 654-660.

14. Yonenaga Y, Mori A, Onodera H, et al. Absence of smooth muscle actin-positive pericyte coverage of tumor vessels correlates with hematogenous metastasis and prognosis of colorectal cancer patients. Oncology 2005;69:159-166.

15. Staton CA, Chetwood AS, Cameron IC, Cross SS, Brown NJ, Reed MW. The angiogenic switch occurs at the adenoma stage of the adenoma carcinoma sequence in colorectal cancer. Gut 2007;56:1426-1432.

16. Des Guetz G, Uzzan B, Nicolas P, et al. Microvessel density 
and VEGF expression are prognostic factors in colorectal cancer. Meta-analysis of the literature. Br J Cancer 2006;94:18231832.

17. Väyrynen SA, Väyrynen JP, Klintrup K, et al. Clinical impact and network of determinants of tumour necrosis in colorectal cancer. Br J Cancer 2016;114:1334-1342.

18. Shimoyama T, Fukuda Y, Kusano H, et al. Microangiographic study of colorectal polyp. J Jpn Soc Coloproctol 1989;42:76-86.

19. Hisabe T, Yao K, Imamura K, et al. White opaque substance visualized using magnifying endoscopy with narrow-band imaging in colorectal epithelial neoplasms. Dig Dis Sci 2014;
59:2544-2549.

20. Mori Y, Kudo SE, Wakamura K, et al. Novel computer-aided diagnostic system for colorectal lesions by using endocytoscopy (with videos). Gastrointest Endosc 2015;81:621-629.

21. Misawa M, Kudo SE, Mori Y, et al. Characterization of colorectal lesions using a computer-aided diagnostic system for narrow-band imaging endocytoscopy. Gastroenterology 2016; 150:1531-1532.

22. Chen PJ, Lin MC, Lai MJ, Lin JC, Lu HH, Tseng VS. Accurate classification of diminutive colorectal polyps using computeraided analysis. Gastroenterology 2018;154:568-575. 